\title{
ОСОБЕННОСТИ ИСПОЛЬЗОВАНИЯ RFID-TЕХНОЛОГИЙ ДЛЯ АВТОМАТИЗАЦИИ ПРОМЫШЛЕННЫХ ПРОЦЕССОВ
}

\section{FEATURES OF USING RFID TECHNOLOGIES FOR INDUSTRIAL PROCESS AUTOMATION}

A. Karyakin

Summary. The article is devoted to the consideration of the features of using RFID technology for the automation of industrial processes. In the course of the research, an example of automation of a direct production process was considered and a mathematical model was proposed for choosing the element base of a radio frequency identification system for objects.

Keywords: industrial process, automation, RFID technology.

\author{
Карякин Александр Тимофеевич \\ К.т.н., дочент, Кабардино-Балкарский \\ государственный университет им. Х. М. Бербекова \\ karyakin2279@mail.ru
}

Аннотация. Статья посвящена рассмотрению особенностей использования RFID-технологий для автоматизации промышленных процессов. В процессе исследования рассмотрен пример автоматизации производственного процесса доставки материалов со склада и запуска цепочки изготовления продукции, а также предложена математическая модель выбора элементной базы для системы радиочастотной идентификации объектов.

Ключевые слова: промышленный процесс, автоматизация, RFID-технология.
B цифровой экономике XXI века информационно-коммуникационные ресурсы приобрели такую же важность и значимость, как сырье, производственное оборудование, трудовой потенциал. Стремительное развитие ИКТ в последние годы повлекло за собой широкомасштабную информационную революцию, которая способствовала повсеместному внедрению автоматизированных систем управления. В настоящее время уже не подлежит сомнению тот факт, что только те производственные предприятия, которые внедрили новые информационные технологии в свои технологические процессы, могут быть конкурентоспособными в современных экономических условиях.

В 2008 году Национальный разведывательный совет США опубликовал отчет, в котором указал на шесть перспективных технологий, которые обладают в обозримой перспективе наибольшей для общества и промышленности «взрывной силой» [1]. Среди этих технологий авторы указывают на технологию радиочастотной идентификации (Radio Frequency IDentification), которая наиболее полно отвечает всем требованиям компьютерной системы управления, где требуется распознавание и регистрация объектов и прав пользователей в реальном масштабе времени. Радиочастотная идентификация - это современный инструмент, с помощью которого можно оптимизировать производственные процессы, а именно автоматизировать процесс сбора и обработки информации бесконтактным способом. Радиочастотная идентификация позволяет автоматически распознавать объекты без непосредственного контакта с ними. При использовании этой технологии с помощью радиосигналов считываются ранее записанные данные, хранящиеся в так называемых RFID-метках [2].

Чаще всего системы радиочастотной идентификации используются в таких сферах, как логистика, торговля, системы контроля и управлением доступом, почта, сельское хозяйство и другие.

Система управления перемещением продукции на базе RFID позволяет оптимизировать транспортные и складские процессы, свести к минимуму влияние человеческого фактора, сократить временные потери, а также утрату товара при его транспортировке. Использование этой технологии в розничной торговле дает возможность не только поддерживать необходимое количество запасов на складе, но и существенно ускорить проведение кассовых расчетов.

В тоже время, современное состояние исследований применения технологии RFID в промышленности характеризуется частичными результатами, полученными в процессе решения локальных задач, которые возникли в работе отдельных предприятий. Это в свою очередь предопределяет необходимость обобщения накопленного опыта в формировании универсальных информационных систем управления промышленными предприятиями с использованием возможностей технологии RFID, что и обуславливает выбор темы данной статьи.

Принимая во внимание широкое распространение RFID-технологий, особенностям их использования в раз- 


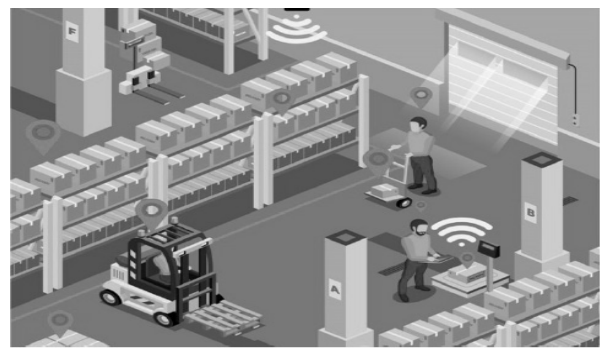

Pис. 1. Пример комплексной системы автоматизации производства на базе RFID-технологии

личных сферах деятельности посвящают свои труды как отечественные, так и зарубежные авторы, в частности, Bergquist Bjarne, Linley John Hastewell, Tao Feng, Никитин А.Б., Манаков А.Д., Болтаев С.Т. и др.

Разработкой модели информационного обмена в процессе управления промышленным производством с использованием RFID-технологий занимаются Лесникова Э.П., Санталова М.С., Нечаева С.Н., Sivasankar V., Buffi Alice и др.

Ведущий инженер АО «АВТОВАЗ» В.В. Королев в статье «Использование RFID меток для построения гибких производственных систем в машиностроении» делает вывод о том, что к числу основных преимуществ данного метода можно отнести следующие: снижение затрат на реализацию системы управления производственным комплексом, надежность хранения информации на RFID метке.

Перспективы использования технологии RFID в промышленности с точки зрения возможности автоматизированного отслеживания времени профилактической проверки и технического обслуживания каждой единицы оборудования, а также контроля степени износа детально описаны в трудах Ильиной Т. А., Кириной Д.Н., Hassan Anas, Bruining Hans, Musa Tagwa и др.

В тоже время, несмотря на значительное количество научных трудов и наработок, посвященных рассматриваемой тематике в настоящее время, необходимо отметить, что в контексте развития Четвертой промышленной революции потенциал использования RFID-технологии только расширяется и соответственно возможности и перспективы их дальнейшей реализации в промышленном секторе, на производственных линиях требуют более углубленных исследований и анализа.

Таким образом, цель статьи заключается в изучении особенностей использования RFID-технологий для автоматизации промышленных процессов.

Как следует из обзора исследований и публикаций, значительный потенциал RFID-технологии для промыш- ленного предприятия заключается в информационном обеспечении управления различными бизнес-процессами, к числу которых относятся:

- учет материально-технических ценностей и инвентаризация;

- идентификация транспорта на предприятии;

- автоматизация активностей персонала и сбор статистики о посетителях;

- защита бренда, фирменной упаковки, контроль сети дистрибуции;

- отслеживание и позиционирование техники в режиме реального времени;

- системы контроля доступа в помещение, к рабочим местам;

- автоматизация производственных процессов.

В тоже время необходимо обратить внимание на тот факт, что технология RFID является вспомогательным средством, которое не может быть самостоятельным инструментом автоматизации деятельности промышленного предприятия, и поэтому должна быть интегрирована в корпоративную информационную систему.

Принимая во внимание тот факт, что традиционные бизнес-процессы (бухгалтерский, складской учет, логистика, контроль за посетителями) характерны для предприятий различных отраслей, с целью выявления специфики использования RFID-технологии именно на промышленном предприятии представляется целесообразным рассмотреть особенности автоматизации производственных процессов.

Обычно загрузка производственной линии начинается с того, что заказ отправляется с сервера компании в производственный цех, затем распечатывается в бумажной форме, чтобы оператор мог знать, какие детали должны быть доставлены для начала работы. При использовании RFID-меток, встроенных в приспособление, такое как лоток, например, или поддон отпадает необходимость распечатки заказа на бумаге. Информация о запросе на комплектующие или сырье отправляется непосредственно на метку и лоток с его содержимым автоматически доставляется к месту отбо- 


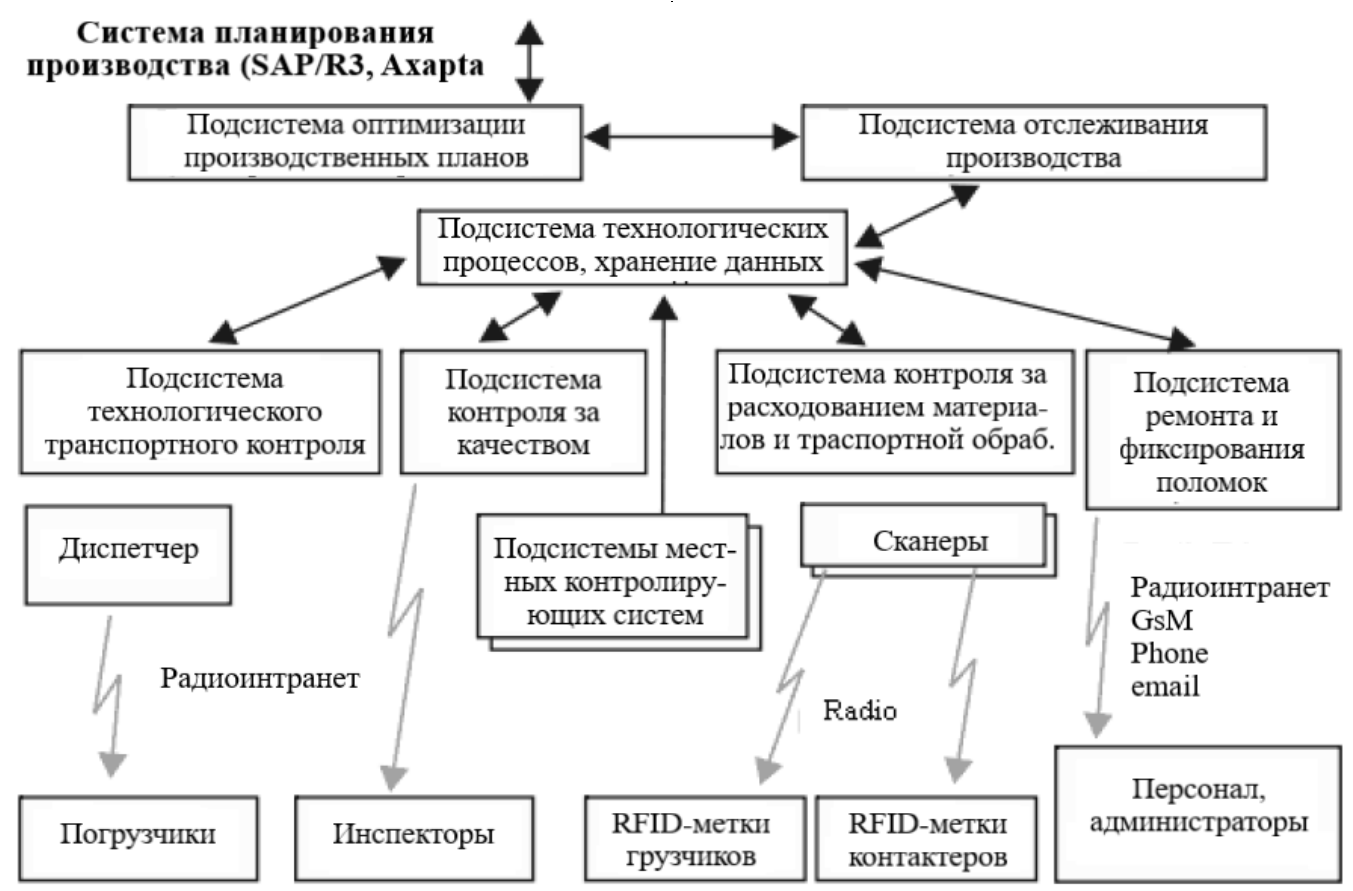

Рис. 2. Уровень автоматизации производства - MES с использованием RFID-технологии

ра первой детали на автоматизированном конвейере или в системе доставки. Это делает процесс безбумажным, сокращая производственные затраты и уменьшая воздействие на окружающую среду (см. рис. 1).

Рассмотрим более подробно пример отслеживания подвижных объектов в производстве с использованием RFID-технологии, которая интегрирована в систему планирования EPR (enterprise resource planning управление ресурсами предприятия), где MES (manufacturing execution system, система управления производством) является связующим звеном между ERP и АСУTП (автоматизированная система управления технологическим процессом).

Процесс формирования заказа для запуска производственной линии с последующем отслеживанием и идентификацией подвижных объектов состоит из следующих этапов: на вход цеха подается сырье со складов предприятия; в цех подаются энергоресурсы (пар, газ, вода, электричество) и выдается заказ на производство продукции; сырье взвешивается и поступает в цех; происходит технологическая обработка; товар поступает на склад готовой продукции.

Процессы, происходящие на уровне автоматизации производства MES представлены на рисунке 2.

Основным этапом проектирования системы радиочастотной идентификации в процессе автоматизации производственных цепочек является выбор технических и программных средств. Эта задача может быть решена методами многокритериальной оптимизации данного типа объектов.

Постановка задачи исследования заключается в следующем.

Пусть будет известно:

- множество объектов MES - систем, которые необходимо отслеживать и идентифицировать;

- множество технических средств элементной базы, которые входят в RFID -системы идентификации;

- параметры и характеристики технических средств;

- множество программных средств, которые характеризуются набором определенных приложений;

- параметры и характеристики приложений, удовлетворяющие выбранным техническим средствам.

Необходимо сформировать элементную базу системы идентификации, которая повысит эффективность и качество проведения всех операций по движению объектов MES - систем.

В состав элементной базы RFID-системы входит считыватель и радиочастотная метка, поэтому выбор технических средств осуществляется путем декомпозиции общего задания на частные. 
Итак, постановка задачи выбора метки заключается в следующем.

Известно, что $D=\left\{D_{i}\right\},(i=\overline{1, i})-$ множество разновидностей меток, где $i-$ число меток, параметры и характеристики меток.

Выбор метки выполняется по следующим функциональным и расходным показателям: рабочая частота метки; радиус считывания; объем памяти, реализация антиколизии, стоимость метки.

Частными критериями оптимизации выбора метки могут быть выбраны следующие:

1. Максимальная рабочая частота метки

$$
R=\max \sum_{i=1}^{i} R \cdot x_{i}
$$

где $x_{i}$ - булева переменная, которая равная 1 , если выбрана $i$-я метка и 0, если $i$-я метка не выбрана.

2. Максимальный радиус считывания

$$
Y=\max \sum_{i=1}^{i} Y \cdot x_{i} \text {. }
$$

\section{3. Максимальный объем памяти}

$$
0=\max \sum_{i=1}^{i} 0 \cdot x_{i}
$$

4. Максимальная антиколизия (возможность выборочного чтения информации из множества меток, которые попали в зону действия считывателя)

$$
A=\max \sum_{i=1}^{i} A M \cdot x_{i} .
$$

\section{5. Минимальная стоимость метки}

$$
B=\min \sum_{i=1}^{i} B M \cdot x_{i}
$$

Область допустимых решений определяется следующими ограничениями: рабочая частота должна быть не менее заданной; радиус считывания должен быть не менее заданного; объем памяти должен быть не менее заданного; антиколизия должна быть не менее заданной; стоимость метки должна быть не более заданной.

Постановка задачи выбора считывателя заключается в следующем. Известно:

множество разновидностей считывателей

$N=\left\{N_{j}\right\},(j=\overline{1, j})$,

где $j$ - число считывателей;

- параметры и характеристики считывателей.

Выбор считывателя выполняется в соответствии со следующими функциональным и расходными показателями: рабочая частота считывателя, радиус считывания, питание, масса, стоимость считывателя.

Таким образом, приведенная математическая модель выбора технических средств радиочастотной идентификации для автоматизации производственного процесса относится к классу задач многокритериальной оптимизации дискретного программирования с булевыми переменными.

Подводя итоги, проведенного исследования можно сделать следующие выводы.

Использование RFID-технологии для автоматизации промышленных процессов позволяет обеспечить: оперативность и наглядность графического представления траекторий движения людей и предметов; разработать гибкую систему правил поведения объектов; обеспечить оперативное оповещение обо всех отклонениях от установленных правил; использовать накопленные данные для ретроспективного анализа с целью их интеграции с существующими системами автоматизации. Предложенная в статье математическая модель выбора технических средств RFID-системы позволит в отличие от существующих, подобрать оптимальный комплект технических средств радиочастотной идентификации по заданным критериям и ограничениями, что значительно уменьшит время на запуск производственного процесса и повысит эффективность работы предприятия в целом.

\section{ЛИТЕРАТУРА}

1. Liu, Q. Automated Logistics Management and Distribution Based on RFID Positioning Technology // Telecommunications and radio engineering. 2020. Volume 79: Number 1; pp 17-28.

2. Hema C. Enhancing hardware and software performance in RFID collisions // Cogent engineering. 2020. Volume 7: Issue 1; pp 15-19.

3. Крюкова А.А., Трухина А. Д. Применение RFID-технологий в современном бизнесе // Актуальные вопросы современной экономики. 2019. № 6-2. С. 759-764. 